\title{
Das Expertenkomitee für Reisemedizin
}

\section{Christoph Hatz}

Präsident der Fachgesellschaft Tropen- und Reisemedizin, Schweizerisches Tropeninstitut, Basel, und Institut für Sozialund Präventivmedizin der Universität Zürich
Hinter diesem Namen steckt ein Konzept zur Verbesserung der reisemedizinischen Beratung, das vor bald 30 Jahren initiiert und seitdem kontinuierlich gepflegt und weiterentwickelt worden ist. Im Bestreben um eine einheitliche und evidenzbasierte Information für Überseereisende, vor allem in tropische und subtropische Länder, haben die reisemedizinischen Zentren in Zürich, Basel, Genf, Lausanne, Bern und St. Gallen gemeinsame Empfehlungen definiert. Auch die Fluggesellschaft Swiss sowie die Fachgesellschaft für Allgemeinmedizin tragen als Mitglieder dazu bei, eine klare und einheitliche Information für Schweizer Reisende zu vermitteln. Die Mitglieder des Komitees, arbeiten unentgeltlich und vermeiden so mögliche Interessenkonflikte. Die Empfehlungen werden schriftlich vom Bundesamt für Gesundheit publiziert und wöchentlich im Internet aktualisiert. Sie können unter www.safetravel.ch und www.tropimed.com abgerufen werden.

Diese Anstrengungen haben über die Jahre zu einer messbaren Verbesserung der reisemedizinischen
Beratung in der Schweiz geführt. In einer Vergleichsstudie konnte eindrücklich gezeigt werden, dass Schweizer Ärztinnen und Ärzte signifikant konsistenter beraten als ihre deutschen Kolleginnen und Kollegen, die nicht über ähnliche Informationen verfügten. Nicht zuletzt aus diesem Grund haben sich in den letzten Jahren weitere Opinion Leaders aus Deutschland, Österreich, Frankreich, Holland, Grossbritannien und Schweden dem Expertenkomitee angeschlossen. Unter anderem konnte so eine beinahe identische Empfehlung zum Malariaschutz in der Schweiz, in Deutschland und Österreich erzielt werden.

Obwohl das Expertenkomitee für Reisemedizin keinen offiziellen Status hat, konnten die Mitglieder dieser Gruppe in den letzten 30 Jahren dazu beitragen, dass sich Personen im deutschsprachigen europäischen Raum bei der reisemedizinischen Beratung auf eine konsens- und evidenzbasierte Expertenmeinung stützen und damit ihre Gesundheitsrisiken auf Reisen besser einschätzen und sich davor schützen können.

\section{Die Fachgesellschaft für Tropen- und Reisemedizin}

\section{Stephan Koch}

Vize-Präsident Fachgruppe Tropen- und Reisemedizin

Korrespondenz: Dr. med. Stephan Koch Zentralstrasse $55 \mathrm{a}$ CH-5610 Wohlen Tel. 0566187007

st.koch@spiderweb.ch www.tropenmedizin-fmh.ch
Die Ausrichtung der Schweizerischen Gesellschaft für Tropenmedizin hat sich seit der Gründung vor 30 Jahren stark gewandelt. Die Kerninhalte der Tropenmedizin sind: Epidemiologie, Public Health, Prävention, Diagnostik und Therapie von Tropen - und Reisekrankheiten. Eine verbesserte Diagnostik hilft, infektiöse Krankheiten schneller zu erkennen, zu behandeln und der Weiterverbreitung vorzubeugen. Die Mobilität der Bevölkerung hat während der letzten Jahrzehnte erheblich zugenommen. Daher beschäftigt sich die Fachgesellschaft in der täglichen Praxis heute viel mehr mit reisemedizinischen Präventionsmassnahmen und klinischen Fragestellungen nach Reisen. Ärztinnen und Ärzte mit jahrelanger Auslandserfahrung bieten Beratungen zu Themen rund um die Gesundheit auf Reisen an und stehen den Kollegen in der hausärztlichen Praxis und in den Spitälern als Konsiliarärzte bei der Differenzialdiagnose von Krankheiten nach einer Reise zur Verfügung. Die Impfberatungen konnten dank der Mitarbeit der Tropenmediziner in der Schweiz und anderen europäischen Ländern vereinheitlicht werden, unter anderem in dem oben erwähnten unabhängigen, multinationalen Expertenkomitee für Reisemedizin.

Die Fachgruppe für Tropen- und Reisemedizin fördert die Ausbildung von jungen Ärzten und die Fortbildung der Mitglieder und ist Teil der Schweizerischen Gesellschaft für Tropenmedizin und Parasitologie.

Die Forschung im Bereich der Tropenmedizin befasst sich unter anderem mit den Public Health-Themen Migrationsmedizin, neglected diseases und diseases among neglected people in sogenannten Entwicklungsländern im Sinne eines gerechten Zugangs zur Gesundheitsversorgung, der für diese Gruppen oft unmöglich oder erschwert ist. Es werden deshalb bei Migranten und Sans-papiers viele allgemeinmedizinische und Infektionskrankheiten nicht oder spät erkannt. Mit Netzwerken in Europa und weltweit versucht die Gesellschaft diese Missstände anzugehen. Bewusstseinsbildungsmassnahmen auf politischer Ebene helfen zudem mit, Lösungsansätze zu erarbeiten. 